\section{NÍVEL DE ATIVIDADE FÍSICA E LOMBALGIA ENTRE FUNCIONÁRIOS DE UMA INSTITUIÇÃO DE ENSINO SUPERIOR NO NORDESTE DO BRASIL}

\author{
Physical activity level and back pain among workers of a \\ higher education institution in Northeast of Brazil
}

\section{RESUMO}

Objetivo: Verificar a associação entre o nível de atividade física habitual e a prevalência de lombalgia entre profissionais de uma instituição de ensino superior em Jequié-BA, Brasil. Métodos: Estudo transversal, censitário, que incluiu 83 dos 102 funcionários da Universidade Estadual do Sudoeste da Bahia - Campus Jequié, obtendo uma taxa resposta de 81,37\%. Utilizou-se um instrumento de coleta de dados contendo oito blocos de informações, nos quais foram incluídas variáveis sociodemográficas, de atividade física habitual e sintomas musculoesqueléticos (dor lombar). A coleta de dados foi realizada por meio de entrevista individual no setor de trabalho do participante. Os dados foram tabulados com EpiData, versão 3.5 e analisados através do programa estatístico Statistical Package for Social Sciences - SPSS, versão 9.0. Resultados: Entre os funcionários, 41 (50,6\%) são ativos fisicamente (praticam pelo menos 150 minutos/semana de atividades físicas). A prevalência de lombalgia nos últimos doze meses foi de 48,1\%. Observou-se que 22 (59,5\%) funcionários considerados fisicamente ativos apresentaram mais queixas de dores lombares quando comparados com os 15 (40,5\%) funcionários inativos. Apesar da presença de lombalgia entre os indivíduos ativos ter sido maior, entende-se que o percentual de dores lombares entre os inativos fisicamente também foi significante. Conclusão: Não foi encontrada associação estatisticamente significante entre o nível de atividade física habitual e a presença de dores lombares.

Descritores: Dor Lombar; Atividade Motora; Saúde do Trabalhador.

\section{ABSTRACT}

Objective: To investigate the association between level of physical activity and prevalence of low back pain among employees of a higher education institution in Jequie-BA, Brazil. Methods: A cross-sectional census study, which included 83 of the 102 employees of the State University of Southwest Bahia, Jequié-Campus, obtaining a response rate of $81.37 \%$. We used a data collection instrument containing eight blocks of information, in which were included social-demographic variables, habitual physical activity and musculoskeletal symptoms (back pain). Data collection was conducted through individual interviews at the working sector of the participant. Data were tabulated with EpiData, version 3.5 and analyzed using Statistical Package for Social Sciences - SPSS, version 9.0. Results: Among employees, $50.6 \%$ were physically active (practicing at least 150 minutes per week of physical activity). The prevalence of low back pain in the last twelve months was $48.1 \%$. We observed that $22(59.5 \%)$ employees considered physically active presented more complaints of back pain compared with 15 (40.5\%) who were inactive. Despite the presence of low back pain among active individuals has shown to be higher, we understand that the percentage of back pain among physically inactive was also significant. Conclusion: There was no statistical significant association between the level of physical activity and the presence of back pain.

Descriptors: Low Back Pain; Motor Activity; Occupational Health.
Artigo Original
1) Universidade Estadual do Sudoeste da Bahia - UESB - Jequié (BA) - Brasil
Recebido em: 26/09/2010 Revisado em: 27/04/2011 Aceito em: 19/05/2011 


\section{INTRODUÇÃO}

O trabalho, enquanto "ação e realização humana", vem mudando seu perfil ao longo dos anos, passando desde uma relação de imposição e servidão a uma condição de moeda de troca, imprescindível à subsistência humana ${ }^{(1)}$. O trabalho para a sociedade moderna pode ter tanto um lado positivo, quando relacionado à construção de bens materiais, sustento da família, realização profissional, construção da identidade, assim como pode gerar condições patológicas quando associado à exploração, insatisfação, sobrecargas e sofrimento $^{(2)}$.

No contexto atual, as características ocupacionais e o próprio ato de produção do trabalho podem refletir, de alguma forma, na saúde do trabalhador. Assim, tanto o trabalhador que pertence a cargos administrativos como aqueles envolvidos em trabalhos braçais estão expostos a influências negativas em sua saúde ${ }^{(3)}$.

Dentre os diversos motivos de afastamento decorrentes de processos patológicos desenvolvidos em determinados ambientes e condições de trabalho destaca-se a lombalgia, que é caracterizada por uma condição de dor na região lombar. Essa patologia aparece como uma das queixas mais registradas, resultando em perdas de dias de trabalho e até mesmo aposentadoria precoce por invalidez $z^{(4-7)}$.

Estima-se que, em alguma fase da vida, cerca de $80 \%$ das pessoas terão dor lombar, sendo uma importante causa de incapacidade e influenciando na qualidade de vida das pessoas $^{(8,9)}$.

Apesar do Brasil ainda não dispor de uma base de dados em que se possa registrar todas essas ocorrências de lombalgia, diversos estudos têm demonstrado que parcela significante da população adulta sofre de dor lombar, acarretando um grande impacto social e econômico para a saúde pública ${ }^{(5,7,10)}$. Em estudos realizados com diversas categorias profissionais, entre as queixas musculoesqueléticas, a região mais afetada é a coluna ${ }^{(11-14)}$.

A atividade física pode ser compreendida como qualquer movimento corporal que demande gasto energético acima dos níveis de repouso, pode ser vivenciada nas dimensões do lazer, transporte, prática esportiva, atividades domésticas e atividades ocupacionais ${ }^{(15)}$. A ausência de realização de algum tipo de atividade física pelos trabalhadores pode constituir um fator de risco para o desenvolvimento e/ou agravamento de diversas condições patológicas, além de aumentar a prevalência da percepção negativa da própria saúde.

Essa realidade foi encontrada em estudos realizados com populações distintas como industriários de Santa Catarina ${ }^{(16,17)}$; em participantes do projeto MONIT
(Monitoramento de Doenças Cardiovasculares e do Diabetes no Brasil) da cidade de Salvador ${ }^{(18)}$ e na população da cidade de Goiânia - GO $^{(19)}$.

Tendo em vista tais evidências, o que se tem percebido é uma mobilização no sentido de incentivar a prática de atividades físicas entre os trabalhadores, tendo em vista o fato de que os benefícios de uma vida ativa para a saúde são inúmeros e cientificamente comprovados ${ }^{(3)}$.

Diante disto, o propósito deste estudo é verificar a associação entre o nível de atividade física habitual e a prevalência de lombalgia entre funcionários da Universidade Estadual do Sudoeste da Bahia.

\section{MÉTODOS}

Estudo epidemiológico de corte transversal, conduzido no período de março a novembro de 2009, com funcionários da Universidade Estadual do Sudoeste da Bahia, Campus de Jequié - BA. Os estudos de corte transversal caracterizamse pela avaliação simultânea de exposição e doença em um ponto do tempo ou num período curto de tempo ${ }^{(20)}$.

A população do estudo compreendeu todos os trabalhadores $(\mathrm{n}=102)$ que possuíam vínculo na Instituição através de contrato temporário, prestação de serviços ou efetivos. Da população do estudo foram excluídos funcionários que se recusaram em participar da pesquisa, não foram encontrados no setor de trabalho após a terceira tentativa e/ou estavam afastados por doença. Após essa etapa a população do estudo compreendeu 83 funcionários (taxa resposta $=81,37 \%$ ), caracterizando um censo.

Utilizou-se instrumento de coleta de dados padronizado, composto por 9 blocos de questões referentes à: características sociodemográficas (sexo, idade, estado civil, nível de escolaridade, renda mensal, raça/ cor); características do trabalho (tempo de trabalho, carga horária semanal, vínculo de trabalho, turno de trabalho, outra atividade remunerada, quantidade de vínculos, posturas corporais); nível de atividade física: avaliado com o Questionário Internacional de Atividades Físicas (IPAC - versão curta) e dor lombar, investigada a partir do Questionário de Sintomas Musculoesqueléticos Nordic musculoskeletal questionnaire ${ }^{(21)}$. Considerouse como "caso" de sintomatologia na região lombar quando o entrevistado referiu esta, nos últimos setes dias, independente do lado afetado.

O IPAC avalia o tempo gasto na realização de atividade física na última semana. As perguntas incluem as atividades praticadas no trabalho, deslocamento, por lazer, por esporte, por exercício ou como parte das suas atividades em casa ou no jardim. Classificou-se como ativos, os indivíduos 
que realizaram 150 minutos ou mais de atividade física por semana, e inativos aqueles que realizaram atividade física com um nível inferior a $150 \mathrm{~min} . / \mathrm{sem}^{(22,23)}$.

Avaliou-se a variável raça/cor por meio do método auto-referido segundo o critério do Instituto Brasileiro de Geografia e Estatística (IBGE), que utiliza categorias de raça/cor: preta, parda, amarela, indígena e branca.

Inicialmente realizou-se o levantamento dos trabalhadores cadastrados que possuíam vínculo na Instituição e os setores aos quais pertenciam. Para coleta de dados realizou-se o treinamento prévio da equipe, sendo que durante a execução da pesquisa todos os setores e os profissionais que ali se encontravam eram convidados a participar.

Os dados foram tabulados com auxilio do software EpiData, versão 3.5, e analisados com o programa estatístico $\mathrm{R}$, versão 2.10.0.

Para análise dos dados procedeu-se à estatística descritiva, incluindo média e desvio-padrão das variáveis contínuas. Quando necessário, as variáveis contínuas foram dicotomizadas, utilizando como ponto de corte suas médias. As variáveis categóricas foram analisadas através da estimativa de frequência (proporções e percentuais). Para avaliar a associação entre as varáveis sociodemograficas, ocupacionais, atividade física e lombalgia aplicou-se, como medida de significância estatística, o teste de Qui-quadrado de Pearson e o teste Exato de Fischer (para valores esperados menores que cinco), adotando $\mathrm{p} \leq 0,05$.

A pesquisa foi aprovada pelo Comitê de Ética em Pesquisa da Universidade Estadual do Sudoeste da Bahia (CEP/UESB), protocolo 008/2009. Os participantes foram esclarecidos sobre os objetivos e procedimentos do trabalho e estiveram livres a qualquer momento em não responder as questões do instrumento de coleta, ou mesmo desistirem de participar da pesquisa.

\section{RESULTADOS}

Na população estudada, $40(50,6 \%)$ dos funcionários era do sexo masculino, $45(57,0 \%)$ com faixa etária de até 38 anos, média de idade de 37,96 $\pm 11,86$ anos. Com relação à escolaridade, 52 (80\%) dos funcionários cursaram nível superior. Observou-se que $44(57,1 \%)$ dos entrevistados afirmaram ser de raça/cor parda, $48(61,5 \%)$ eram casados/ união estável e 45 (57,0\%) possuíam filhos (Tabela I).

O tempo de trabalho dos colaboradores na instituição, em média, foi de 9,70 $\pm 9,24$ anos, com tempo mínimo de trabalho de 1 ano e máximo de 32 anos. As características ocupacionais apresentadas na tabela II demonstram que $54(70,1 \%)$ dos funcionários eram efetivos, $49(61,3 \%)$ possuía tempo na ocupação inferior a 9,7 anos, 61 (79,2\%)
Tabela I - Caracterização da população de trabalhadores, segundo variáveis sociodemográficas. Jequié-BA, 2009.

\begin{tabular}{lrr}
\hline Variáveis (N*) & $\mathbf{n}$ & $\mathbf{\%}$ \\
\hline Sexo (79) & 39 & 49,4 \\
$\quad$ Feminino & 40 & 50,6 \\
$\quad$ Masculino & & \\
Faixa Etária (79) & 45 & 57,0 \\
$\quad$ Até 38 anos & 34 & 43,0 \\
$\quad 38$ ou mais anos & & \\
Escolaridade (65) & 1 & 1,5 \\
$\quad$ Fundamental & 12 & 18,5 \\
$\quad$ Ensino médio & 52 & 80,0 \\
$\quad$ Superior & & \\
Cor da pele/Raça (77) & 11 & 14,3 \\
$\quad$ Negra & 44 & 57,1 \\
$\quad$ Parda & 1 & 1,3 \\
$\quad$ Amarela & 19 & 24,7 \\
$\quad$ Branca & & \\
Situação Conjugal (78) & 48 & 61,5 \\
$\quad$ Casados/união estável & 30 & 38,5 \\
$\quad$ Solteiros/viúvos/separados/divorciados & & \\
Filhos (79) & 45 & 57,0 \\
$\quad$ Sim & 34 & 43,0 \\
$\quad$ Não &
\end{tabular}

*Estas variáveis não foram preenchidas em parte dos questionários analisados no estudo, por isso apresentam um $n$ inferior ao número total estudado.

do turno diurno fixo de trabalho e $40(51,3 \%)$ já trabalhou em outro setor, $47(60,3 \%)$ dos entrevistados não possuía outra atividade remunerada, $67(82,7 \%)$ exercia seu trabalho frequentemente na postura sentada e $66(79,5 \%)$ não permaneciam em postura forçada.

Entre os entrevistados, 41 (50,6\%) foram considerados ativos e $40(49,4 \%)$ inativos fisicamente. Os funcionários inativos fisicamente foram principalmente mulheres (26$66,7 \%)$, com idade inferior a 40 anos $(24-51,1 \%)$, casados/ união estável (28 - 58,3\%), menor nível de escolaridade (07 $-53,8 \%)$, com filhos $(25-55,6 \%)$ e faixa salarial de quatro ou mais salários mínimos (SM) $(25$ - 48,1 \%) (Tabela III).

O nível de atividade física, segundo características do trabalho, evidenciou que os funcionários que ocupavam cargos administrativos $(32-48,5 \%)$ possuíam maior tempo de trabalho $(34-50,7 \%)$, com carga horária de 20 horas semanais $(5-55,6 \%)$ e turno diurno de trabalho (35$52,5 \%$ ) eram menos ativos fisicamente. Entretanto, esses resultados não foram estatisticamente significantes (Tabela III).

A prevalência de dor lombar nos funcionários nos últimos doze meses foi de $48,1 \%$ e nos últimos sete dias foi de $28,6 \%$. Observou-se que indivíduos com idade 
Tabela II - Caracterização da população de trabalhadores, segundo variáveis ocupacionais. Jequié-BA, 2009.

\begin{tabular}{lrr}
\hline Variáveis (N*) & n & \% \\
\hline Vínculo de trabalho (77) & & \\
$\quad$ Contrato temporário & 10 & 13,0 \\
$\quad$ Prestação de serviços & 13 & 16,9 \\
$\quad$ Efetivo & 54 & 70,1 \\
Tempo de trabalho (80) & & \\
$\quad$ Até 9,7 anos & 49 & 61,3 \\
$\quad$ 9,7 ou mais anos & 31 & 38,8 \\
Turno de trabalho (77) & & \\
$\quad$ Diurno fixo & 61 & 79,2 \\
$\quad$ Noturno fixo & 4 & 5,2 \\
$\quad$ Diurno/noturno alternante & 7 & 9,1 \\
$\quad$ Revezamento de turno & 5 & 6,5 \\
Trabalhou em outro setor (78) & & \\
$\quad$ Sim & 40 & 51,3 \\
$\quad$ Não & 38 & 48,7 \\
Possui outra atividade remunerada (78) & & \\
$\quad$ Sim & 31 & 39,7 \\
$\quad$ Não & 47 & 60,3 \\
Postura sentada (81) & & \\
$\quad$ Frequente & 67 & 82,7 \\
$\quad$ Pouco frequente & 14 & 17,3 \\
Permanência em postura forçada (80) & & \\
$\quad$ Frequente & 14 & 16,9 \\
Pouco frequente & 66 & 79,5 \\
\hline
\end{tabular}

*Estas variáveis não foram preenchidas em parte dos questionários analisados no estudo, por isso apresentam um $\mathrm{n}$ inferior ao número total estudado.

menor que 38 anos (23 - 51,1\%), casados/união estável ( $26-54,2 \%)$, nível superior de escolaridade $(28-53,8 \%)$, filhos $(22-48,9 \%)$ e número de filhos de dois ou mais ( 15 $50,0 \%$ ) apresentaram uma maior prevalência de dor lombar (Tabela IV).

Os entrevistados com tempo de trabalho superior a 9,7 anos $(24-49,0 \%)$, carga horária de 40 ou mais horas de trabalho semanais $(4-66,7 \%)$ e dois ou mais vínculos empregatícios (4-66,7\%) apresentaram maior prevalência de dor lombar (Tabela IV).

Ao analisar a associação entre o nível de atividade física e lombalgia observou-se que a prevalência de dor lombar foi maior entre funcionários considerados fisicamente ativos, contudo a associação não foi estatisticamente significante (Tabela V).

\section{DISCUSSÃO}

Os resultados encontrados neste estudo revelaram o perfil da população de trabalhadores, no qual houve maior proporção de funcionários do sexo masculino na instituição. Este fato revela que ainda há resistência por parte da sociedade em atribuir às mulheres cargos que não sejam relacionados à educação, à saúde, entre outras áreas estigmatizadas como de caráter feminino.

Uma parcela significativa da população estudada foi considerada fisicamente inativa. Quando analisada a distribuição do nível de atividade física por sexo, os resultados corroboram os achados de outros levantamentos realizados ${ }^{(16,18,19)}$. Esse achado tem sido comumente observado na literatura e pode ser explicado pelas múltiplas jornadas de trabalho das mulheres da sociedade moderna, já que tendem a dividir o tempo entre trabalho e o cuidado da casa e dos filhos ${ }^{(24)}$.

Em relação à associação positiva entre presença de um companheiro e maior frequência de inatividade física, encontrada no presente estudo, também houve semelhança com as evidências apresentadas em estudo com industriários de Santa Catarina ${ }^{(16)}$ e universitários do Rio de Janeiro ${ }^{(25)}$.

Entretanto, resultado inverso foi encontrado no estudo realizado com a população adulta de Goiânia, no qual se observou uma frequência maior de sedentarismo entre as mulheres sem companheiros ${ }^{(19)}$.

Em relação à dor lombar, esta foi referida por um número considerável de trabalhadores, corroborando com estudos realizados com outras categorias profissionais, nos quais a coluna foi a região mais afetada ${ }^{(5,11,19,22)}$.

Esse quadro já era esperado, tendo em vista que a população estudada trabalha frequentemente em postura sentada. A realização do trabalho em postura sentada por longos períodos, apesar de ser mais confortável, gera mais danos à estrutura da coluna lombar, uma vez que provoca o aumento da pressão nos discos intervertebrais ${ }^{(6)}$.

Da mesma forma, outros autores consideram a postura hipocinética como um dos fatores que desestabilizam as estruturas osteoligamentares e musculares que contribuem para o equilíbrio da coluna lombar, gerando assim a instabilidade do complexo lombo-pélvico e, consequentemente, quadros dolorosos e lesões na região lombar ${ }^{(26,27)}$.

A coluna é uma região de extrema importância para o trabalho, por servir de sustentação do corpo e rotação do tronco, sendo muitas vezes utilizada de forma 
Tabela III - Características sociodemográficas e ocupacionais dos trabalhadores, segundo nível de atividade física. JequiéBA, 2009.

\begin{tabular}{|c|c|c|c|}
\hline \multirow[b]{2}{*}{ Variáveis } & \multicolumn{2}{|c|}{ Nível de Atividade Física } & \multirow[b]{2}{*}{$p$} \\
\hline & $\begin{array}{l}\text { Ativos } \\
\text { n (\%) }\end{array}$ & $\begin{array}{c}\text { Inativos } \\
\text { n (\%) }\end{array}$ & \\
\hline Sexo & & & 0,01 \\
\hline Masculino & $28(70,0)$ & $12(30,0)$ & \\
\hline Feminino & $13(33,3)$ & $26(66,7)$ & \\
\hline Faixa Etária & & & 0,52 \\
\hline Até $39 \mathrm{E}>4024<3 \mathrm{SM}$ anos & $23(48,9)$ & $24(51,1)$ & \\
\hline 40 ou mais anos $24<3 \mathrm{SM} \mathrm{la}>10$ anos ab $>20$ & $18(56,3)$ & $14(43,8)$ & \\
\hline Situação Conjugal & & & 0,02 \\
\hline Casados/união estável & $20(41,7)$ & $28(58,3)$ & \\
\hline Solteiros/viúvos/separados/divorciados & $21(67,7)$ & $10(32,3)$ & \\
\hline Escolaridade & & & 0,62 \\
\hline Ensino Superior & $28(53,8)$ & $24(46,2)$ & \\
\hline Ensino Médio & $6(46,2)$ & $7(53,8)$ & \\
\hline Filhos & & & 0,13 \\
\hline Não & $21(61,8)$ & $13(38,2)$ & \\
\hline Sim & $20(44,4)$ & $25(55,6)$ & \\
\hline Faixa Salarial & & & 0,73 \\
\hline Até $3 \mathrm{SM}$ la $>10$ anos $\mathrm{ab}>20 \mathrm{~h}$ rá & $8(57,1)$ & $6(42,9)$ & \\
\hline 4 ou mais $\mathrm{SM}$ & $27(51,9)$ & $25(48,1)$ & \\
\hline Ocupação & & & $1,00^{*}$ \\
\hline Administrativo & $34(51,5)$ & $32(48,5)$ & \\
\hline Serviços Gerais & $6(54,5)$ & $5(45,5)$ & \\
\hline Tempo de Trabalho & & & 0,59 \\
\hline Até 1 ano $a b>20 \mathrm{~h}$ rá & $8(57,1)$ & $6(42,9)$ & \\
\hline 2 ou mais anos & $33(49,3)$ & $34(50,7)$ & \\
\hline Carga Horária Semanal & & & $0,73^{*}$ \\
\hline 20 horas rá & $44,4(4)$ & $5(55,6)$ & \\
\hline 40 ou mais horas & $52,9(36)$ & $32(47,1)$ & \\
\hline Turno de Trabalho & & & $0,34^{*}$ \\
\hline Diurno & $47,5(29)$ & $32(52,5)$ & \\
\hline Noturno & $50,0(2)$ & $2(50,0)$ & \\
\hline Alternante & $66,7(8)$ & $4(3,33)$ & \\
\hline
\end{tabular}

* Fischer's Exact.

inadequada pela má postura e carregamento de pesos, o que consequentemente pode acarretar a lombalgia ${ }^{(5,28)}$.

Esse quadro pode ser considerado como reflexo das mudanças ocorridas na sociedade. Se por um lado os avanços tecnológicos têm facilitado a vida das pessoas em diversos sentidos, por outro têm contribuído para a formação de uma sociedade que se movimenta cada vez menos e que vem adotando posturas inadequadas para o equilíbrio das estruturas da coluna vertebral ${ }^{(29,30)}$.

De acordo com a literatura atual, a dor lombar costuma atingir a população com menos de 45 anos, sendo um dos motivos mais frequentes de limitações físicas e aposentadorias por invalidez no $\mathrm{Brasil}^{(10)}$. Os resultados encontrados neste estudo mostraram que essa afirmativa é 
Tabela IV - Características sociodemográficas e ocupacionais dos trabalhadores, segundo sintomatologia musculoesquelética na região lombar. Jequié-BA, 2009.

\begin{tabular}{|c|c|c|c|}
\hline \multirow[b]{2}{*}{ Variáveis } & \multicolumn{2}{|c|}{ Dor Lombar (nos últimos 12 meses) } & \multirow[b]{2}{*}{$p$} \\
\hline & $\begin{array}{c}\text { Sim } \\
\text { n (\%) }\end{array}$ & $\begin{array}{c}\text { Não } \\
\text { n (\%) }\end{array}$ & \\
\hline Faixa Etária & & & 0,53 \\
\hline Menor que $38 \mathrm{E}>4024<3 \mathrm{SM}$ anos & $15(41,1)$ & $19(55,9)$ & \\
\hline 38 ou mais anos $24<3$ SMla $>10$ anos ab $>20$ & $23(51,1)$ & $22(48,9)$ & \\
\hline Situação Conjugal & & & 0,22 \\
\hline Casados/união estável & $26(54,2)$ & $22(45,8)$ & \\
\hline Solteiros/viúvos/separados/divorciados & $12(40,0)$ & $18(60,0)$ & \\
\hline Escolaridade & & & 0,06 \\
\hline Ensino Superior & $28(53,8)$ & $24(46,2)$ & \\
\hline Ensino Médio & $3(23,1)$ & $10(76,9)$ & \\
\hline Filhos & & & 0,87 \\
\hline Não & $16(47,1)$ & $18(52,9)$ & \\
\hline Sim & $22(48,9)$ & $23(51,1)$ & \\
\hline Número de Filhos & & & 0,84 \\
\hline Até 1 filhola $>10$ anos $a b>20$ hrá & $8(47,1)$ & $9(52,9)$ & \\
\hline 2 ou mais filhos & $15(50,0)$ & $15(50,0)$ & \\
\hline Tempo de Trabalho & & & 0,95 \\
\hline Menor que 9,7 anos ab> 20 hrá & $15(48,4)$ & $16(51,6)$ & \\
\hline 9,7 ou mais anos & $24(49,0)$ & $25(51,0)$ & \\
\hline Carga Horária Semanal & & & 0,64 \\
\hline 20 horas rá & $9(50,0)$ & $9(50,0)$ & \\
\hline 40 ou mais horas & $4(66,7)$ & $2(33,3)$ & \\
\hline Outros Vínculos & & & 0,65 \\
\hline 1 vínculo & $10(50,0)$ & $10(50,0)$ & \\
\hline 2 ou mais vínculos & $4(66,7)$ & $2(33,3)$ & \\
\hline
\end{tabular}

Tabela V - Atividade Física e Dor Lombar dos trabalhadores. Jequié-BA, 2009.

\begin{tabular}{lcc}
\hline $\begin{array}{c}\text { Dor Lombar } \\
\text { (últimos 12 meses) }\end{array}$ & $\begin{array}{c}\text { Ativos } \\
\text { n (\%) }\end{array}$ & $\begin{array}{c}\text { Inativos } \\
\text { n (\%) }\end{array}$ \\
\hline Sim & $22(59,5)$ & $15(40,5)$ \\
Não & $19(45,2)$ & $23(54,8)$ \\
\hline
\end{tabular}

Nível de significância $(\mathrm{p}<0,20)$

plausível, dado que entre os funcionários com até 38 anos, o relato de dor lombar foi maior, mesmo não havendo associação estatística. Em outro estudo ${ }^{(31)}$ foi identificado que a média de idade da população com dor lombar foi de 36,7 anos e 37,3 anos $\pm 4,8$ anos, respectivamente.
Em relação ao estado civil, a prevalência de dor lombar foi maior entre os funcionários com companheiros. Da mesma forma os entrevistados que afirmaram ter filhos apresentaram um valor significante de queixas de dores lombares. Essa ocorrência pode ser resultante das demandas encontradas no ambiente familiar e doméstico, já que o trabalho doméstico pode contribuir para a ocorrência do efeito de interesse estudo, ou seja, a dor lombar poderia ser potencializada para aqueles indivíduos que têm atividade na famíliai ${ }^{(32)}$.

A associação entre o nível de escolaridade e a dor lombar neste estudo também foi encontrada em um estudo realizado com acadêmicos e funcionários de um Centro universitário ${ }^{(5)}$. Esses achados confirmam ainda mais a ideia de que fatores psicológicos como estresse, insatisfação, 
angústia, desmotivação e sobrecargas psíquicas, comuns em populações que buscam o incessante aperfeiçoamento profissional numa sociedade competitiva, podem gerar processos dolorosos na região lombar.

$\mathrm{Na}$ associação entre fatores ocupacionais (tempo de trabalho, carga horária e número de vínculos) e a prevalência de lombalgia observou-se que, pessoas com menor tempo de trabalho, menor carga horária e mais vínculos empregatícios apresentaram maiores prevalências de dores lombares, entretanto esses resultados não foram estatisticamente significantes. Esse resultado é o inverso dos achados encontrados em outros estudos ${ }^{(32,33)}$. Em um levantamento realizado com professores, foi verificada uma alta prevalência de sintomas musculoesqueléticos naqueles que possuíam carga horária de 40 horas semanais e maior tempo de trabalho ${ }^{(34)}$.

Não foi encontrada associação estatisticamente significante entre nível de atividade física e a lombalgia nos funcionários da instituição de ensino superior. A literatura atual entende que a imobilização e a inatividade física provocam efeitos deletérios à coluna vertebral, sendo, portanto, recomendável a prática de atividades físicas (principalmente o exercício físico) como uma forma de tratamento e prevenção da lombalgia.

Nos achados deste estudo, observou-se que os funcionários considerados fisicamente ativos apresentam mais queixas de dores lombares se comparados com os funcionários inativos. Apesar desta proporção de quadros álgicos entre os indivíduos ativos ser maior, entende-se que o percentual de dores lombares entre os inativos fisicamente também é significante.

\section{CONCLUSÃO}

Os achados revelaram uma alta prevalência de lombalgia entre os servidores e elevada proporção de inatividade física, tornando-se um problema preocupante da área de saúde do trabalhador. Contudo, não foi encontrada associação estatisticamente significante entre o nível de atividade física habitual e a prevalência de lombalgia.

Os conhecimentos gerados podem se transformar em subsídios para o planejamento de ações de prevenção de queixas de lombalgias através da realização de atividade física e melhoria das condições de trabalho, contribuindo assim para a promoção da saúde e melhoria da qualidade de vida destes funcionários.

É necessária a realização de novos estudos sobre o tema de forma aprofundada, observando outros aspectos que podem estar associados a este agravo, a fim de entender melhor a relação entre atividade física e lombalgia.

\section{REFERÊNCIAS}

1. Muller A, Dacosta L. Lazer e Trabalho: um único ou múltiplos olhares? Santa Cruz do Sul: EDUNISC; 2003.

2. Dejours C. A Loucura do trabalho: estudo da psicopatologia do trabalho. $5^{\text {a }}$ ed. São Paulo: Cortez Oboré; 1992.

3. Matsudo SMM, Matsudo VKR, Andrade DR, Oliveira LC, Araújo TL. Promovendo Atividade Física no Ambiente de Trabalho. Rev Ativ Física e Med Esp. 2007;12(2):97-102.

4. Torres G, Marinho ISF. Causas de lombalgia em grupos de pessoas sedentárias e praticantes de atividades físicas. Revista Digital. 2006;10:92.

5. Domínguez AGD, Alves D, Gomes EP, Ewerton FM. Prevalência de lombalgia em acadêmicos e funcionários do Centro universitário UNIEURO. Rev Elet Fisiot Centro Universitário. 2008;2:63-8.

6. Santos Filho SB, Barreto SM. Atividade ocupacional e prevalência de dor osteomuscular em cirurgiõesdentistas de Belo Horizonte, Minas Gerais, Brasil: contribuição ao debate sobre os distúrbios osteomusculares relacionados ao trabalho. Cad Saúde Pública. 2001; 17(1):181-93.

7. Silva ALR. Correlação entre lombalgia e as características antropométricas de trabalhadores bancários da cidade de Londrina - PR [dissertação]. Santa Catarina: Universidade Federal de Santa Catarina; 1999.

8. Andrade SC, Araújo AGR, Vilar MJP. Escola de Coluna: revisão histórica e sua aplicação na lombalgia crônica. Rev Bras Reumatol. 2005. 45(4):224-8.

9. Ehrlich GE. Low back pain. Bull World Health Organ. 2003; 81:671-6.

10. Costa D, Palma A. O efeito do treinamento contra resistência na síndrome da dor lombar. Rev Port Cien Desp. 2007; 2:224-34.

11. Gurgueira GP, Alexandre NMC, Corrêa Filho HR. Prevalência de sintomas músculo-esqueléticos em trabalhadores de enfermagem. Rev Latinoam Enferm. 2003;11(5):608-13.

12. Carneiro LRV, Coqueiro RS, Freire MO, Barbosa, AR. Sintomas de distúrbios osteomusculares em motoristas e cobradores de ônibus. Rev Bras Cineantropom Desempenho Hum. 2007;9(3):277-83. 
13. Picoloto D, Silveira E. Prevalência de sintomas osteomusculares e fatores associados em trabalhadores de uma indústria metalúrgica de Canoas- RS. Ciênc Saúde Col. 2008;13(2): 507-16.

14. Dávila LS, Sousa GAF, Sampaio RF. Prevalência de desordens musculoesqueléticas relacionadas ao trabalho em fisioterapeutas da rede hospitalar SUS-BH. Rev Bras Fisioterapia. 2005;9(2):219-25.

15. Carpersen CJ, Powell KE, Christensan GM. Physical activity, exercise, and physical activity fitness: definitions and distinctions for health-related research. Public Health Rep. 1985;100(2):126-31.

16. Barros MVG, Nahas MV. Comportamentos de risco, auto-avaliação do nível de saúde e percepção de estresse entre trabalhadores da indústria. Rev Saúde Pública. 2000;35(6): 554-63.

17. Fonseca SA, Blank VLG, Barros MVG, Nahas MV. Percepção de saúde e fatores associados em industriários de Santa Catarina, Brasil. Cad Saúde Pública. 2008;24(3):567-76.

18. Pitanga FJG, Lessa I. Associação entre inatividade física no tempo livre e internações hospitalares em adultos na cidade de Salvador-Brasil. Rev Bras Cineantropom Desempenho Hum. 2008;10(4):347-53.

19. Cunha IC, Peixoto MRG, Jardim PCB, Alexandre VP. Fatores associados à prática de atividade física na população adulta de Goiânia. Rev Bras Epidemiol. 2008;11(3):495-504.

20. Medronho RA. Epidemiologia. $2^{\mathrm{a}}$ ed. São Paulo: Atheneu; 2009.

21. Kuorinka I, Jonsson B, KilbomA, Vinterberg H, BieringSrensen F, Andersson G, et al. Standardised Nordic Questionnaires for the Analysis of Musculoskeletal Symptoms. Appl Ergon. 1987;18(3):233-7.

22. Benedetti TRB, Antunes PC, Rodriguez-Añez CR, Mazo GZ, Petroski EL. Reprodutibilidade e validade do Questionário Internacional de Atividade Física (IPAQ) em homens idosos. Rev Bras Med Esporte. 2007;13(1).

23. Toscano JJO, Oliveira ACC. Qualidade de Vida em Idosos com Distintos Níveis de Atividade Física. Rev Bras Med Esporte. 2009;15(9):169-73.

24. Bagrichevsky M, Estevão A, Vaconsellos-Silva PR. Sedentarismo, nós e o mundo (im)possível no contexto da ciência. Rev Saúde Pública. 2007;41(5):862-4.
25. Salles-Costa R, Heilborn ML, Werneck GL, Faerstein E, Lopes CS. Gênero e prática de atividade física de lazer. Cad Saúde Pública. 2003;19 (Suppl 2):S325-33.

26. Gurgueira GP, Alexandre NMC, Corrêa Filho HR. Prevalência de sintomas músculo-esqueléticos em trabalhadores de enfermagem. Rev Latinoam Enferm. 2003;11(5):608-13.

27. Carneiro LRV, Coqueiro RS, Freire MO, Barbosa, AR. Sintomas de distúrbios osteomusculares em motoristas e cobradores de ônibus. Rev Bras Cineantropom Desempenho Hum. 2007;9(3):277-83.

28. Picoloto D, Silveira E. Prevalência de sintomas osteomusculares e fatores associados em trabalhadores de uma indústria metalúrgica de Canoas- RS. Cien Saúde Col. 2008;13(2):507-16.

29. Cox JM. Dor Lombar: Mecanismo, Diagnostico e Tratamento. 6 ${ }^{\mathrm{a}}$ ed. Manole: São Paulo; 2002.

30. Kleinpaul JF, Mann L. Dor lombar e exercício físico: uma revisão. Revista Digital. 2008;13(127).

31. Brennan GP, Ruhling RO, Hood RS, Shultz BAB, Johnson SC, Andrews BC. Physical Caracteristics of patients with herniated intervertebral lumbar discs. Spin. 1987;12(7): 699-702.

32. Araújo TM, Pinho OS, Almeida MMG. Prevalência de transtornos mentais comuns em mulheres e sua relação com as características sociodemográficas e o trabalho doméstico. Rev Bras Saúde Materno Infantil. 2005;5(3):337-48.

33. Araújo TM, Carvalho FM. Condições de trabalho docente e saúde na Bahia: estudos epidemiológicos. Educ Soc. 2009;30:427-49.

34. Cardoso JP, Ribeiro IQB, Araujo TM, Carvalho FM, Reis EJFB. Prevalência de dor musculoesquelética em professores. Rev Bras Epidemiol. 2009;12(4):604-14.

\section{Endereço de correspondência:}

Ana Valéria Reis Souza

Núcleo de Estudos em Saúde da População

Universidade Estadual do Sudoeste da Bahia (UESB)

Avenida José Moreira Sobrinho, s/n

Bairro: Jequiezinho

CEP: 45206-190 - Jequié - BA - Brasil

E-mail: saulosaudecoletiva@gmail.com 